\title{
Constraint Propagation of $C^{2}$-adjusted Formulation II - Another Recipe for Robust Baumgarte-Shapiro-Shibata-Nakamura Evolution System -
}

\author{
Takuya Tsuchiya* and Gen Yoneda \\ Department of Mathematical Sciences, Waseda University, Okubo, Shinjuku, Tokyo, 169-8555, Japan \\ Hisa-aki Shinkai \\ Faculty of Information Science and Technology, Osaka Institute of Technology, \\ 1-79-1 Kitayama, Hirakata, Osaka 573-0196, Japan \\ Computational Astrophysics Laboratory, Institute of Physical $\& 3$ Chemical \\ Research (RIKEN), Hirosawa, Wako, Saitama, 351-0198 Japan
}

(Dated: December 6, 2018)

\begin{abstract}
In order to obtain an evolution system which is robust against the violation of constraints, we present a new set of evolution systems based on the so-called Baumgarte-Shapiro-Shibata-Nakamura (BSSN) equations. The idea is to add functional derivatives of the norm of constraints, $C^{2}$, to the evolution equations, which was proposed by Fiske (2004) and was applied to the ADM formulation in our previous study. We derive the constraint propagation equations, discuss the behavior of constraint damping, and present the results of numerical tests using the gauge-wave and polarized Gowdy wave spacetimes. The construction of the $C^{2}$-adjusted system is straightforward. However, in BSSN, there are two kinetic constraints and three algebraic constraints; thus, the definition of $C^{2}$ is a matter of concern. By analyzing constraint propagation equations, we conclude that $C^{2}$ should include all the constraints, which is also confirmed numerically. By tuning the parameters, the lifetime of the simulations can be increased as 2-10 times as longer than those of the standard BSSN evolutions.
\end{abstract}

PACS numbers: 04.25.D-

\section{INTRODUCTION}

When solving the Einstein equations numerically, the standard way is to split the spacetime into space and time. The most fundamental decomposition of the Einstein equations is the Arnowitt-Deser-Misner (ADM) formulation [1, 2]. However, it is well known that in longterm evolutions in strong gravitational fields such as the coalescences of binary neutron stars and/or black holes, simulations with the ADM formulation are unstable and are often interrupted before producing physically interesting results. Finding more robust and stable formulations is known to the "formulation problem" in numerical relativity [3 [5].

Many formulations have been proposed in the last two decades. The most commonly used sets of evolution equations among numerical relativists are the so-called Baumgarte-Shapiro-Shibata-Nakamura (BSSN) formulation [6, 7], the generalized harmonic $(\mathrm{GH})$ formulation [8, 9], the Kidder-Scheel-Teukolsky (KST) formulation [10], and the Z4 formulation [11, 12] (as references of their numerical application, we here cite only well-known articles; 13, 14 for the BSSN formulation, 15] for the GH formulation, [16] for the KST formulation, and [17] for the $\mathrm{Z} 4$ formulation).

All of the above modern formulations include the technique of "constraint damping", which attempts to con-

* tsuchiya@akane.waseda.jp trol the violations of constraints by adding the constraint terms to their evolution equations. Using this technique, more stable and accurate systems are obtained (see e.g. [18, 19]). This technique can be described as 'adjustment' of the original system.

In 20 22], two of the authors systematically investigated how the adjusted terms change the original systems by calculating the constraint propagation equations. The authors suggested some effective adjustments for the BSSN formulation under the name "adjusted BSSN formulation" 22]. The actual constraint-damping effect was confirmed by numerical tests [23].

Fiske proposed a method of adjusting the original evolution system using the norm of the constraints, $C^{2},[24$, which we call a " $C^{2}$-adjusted system." The new evolution equations force the constraints to evolve towards their decay if the coefficient parameters of the adjusted terms are set as appropriate positive values. Fiske reported the damping effect of the constraint violations for the Maxwell system 24] and for the linearized ADM and BSSN formulations 25]. He also reported the limitation of the magnitude of the coefficient parameters of the adjusted terms.

In 26], we applied this $C^{2}$-adjusted system to the (full) $\mathrm{ADM}$ formulation and presented some numerical tests. We confirmed that the violations of the constraints are less than those in the original system. We also reported the differences of the effective range of the coefficient of the adjusted terms.

In this article, we apply the $C^{2}$-adjusted system to the 
(full) BSSN formulation and derive the constraint propagation equations in the flat space. We perform some numerical tests and compare them with three other types of BSSN formulations: the standard BSSN formulation, the $\widetilde{A}$-adjusted BSSN formulation, and the $C^{2}$-adjusted BSSN formulation. We use the gauge-wave and polarized Gowdy wave testbeds, which are the test problems as is known to apples-with-apples testbeds for comparing evolution systems [27]. Since the models are precisely fixed up to the gauge conditions, boundary conditions, and technical parameters, the testbeds are widely used for comparisons [23, 28, 29].

The structure of this article is as follows. We review the ideas of adjusted systems and $C^{2}$-adjusted system in Sec II. In Sec III, we review the standard and adjusted BSSN formulations and derive the $C^{2}$-adjusted version of the BSSN formulation. In SecIV we present some numerical tests of the gauge-wave and polarized Gowdy wave testbeds. We show the damping effect of the constraint violations, and confirm that inclusion of algebraic constraints in $C^{2}$ make the violations of constraints decrease. We summarize this article in Sec $\nabla$ In this article, we only consider vacuum spacetime, but the inclusion of matter is straightforward.

\section{IDEAS OF ADJUSTED SYSTEMS AND $C^{2}$-ADJUSTED SYSTEMS}

\section{A. Idea of adjusted systems}

Suppose we have dynamical variables $u^{i}$ that evolve with the evolution equations

$$
\partial_{t} u^{i}=f\left(u^{i}, \partial_{j} u^{i}, \cdots\right),
$$

and suppose also that the system has the (first class) constraint equations

$$
C^{a}\left(u^{i}, \partial_{j} u^{i}, \cdots\right) \approx 0
$$

We can then predict how the constraints are preserved by evaluating the constraint propagation equations

$$
\partial_{t} C^{a}=g\left(C^{a}, \partial_{i} C^{a}, \cdots\right),
$$

which measure the violation behavior of constraints $C^{a}$ in time evolution. Equation (2.3) is theoretically weakly zero, i.e., $\partial_{t} C^{a} \approx 0$, since the system is supposed to be the first class. However, free numerical evolution with discretized grids introduces a constraint violation, at least at the level of truncation error, which sometimes grows and stops the simulations. The unstable feature of ADM evolution can be understood on the basis of this analysis [15].

Such features of the constraint propagation equations, (2.3), change when we modify the original evolution equations. Suppose we add constraint terms to the righthand-side of (2.1) as

$$
\partial_{t} u^{i}=f\left(u^{i}, \partial_{j} u^{i}, \cdots\right)+F\left(C^{a}, \partial_{j} C^{a}, \cdots\right),
$$

where $F\left(C^{a}, \cdots\right) \approx 0$ in principle zero but not exactly zero in numerical evolutions. With this adjustment, equation (2.3) will also be modified to

$$
\partial_{t} C^{a}=g\left(C^{a}, \partial_{i} C^{a}, \cdots\right)+G\left(C^{a}, \partial_{i} C^{a}, \cdots\right) .
$$

Therefore, we are able to control $\partial_{t} C^{a}$ by making an appropriate adjustment $F\left(C^{a}, \partial_{j} C^{a}, \cdots\right)$ in (2.4). If $\partial_{t} C^{a}<0$ is realized, then the system has the constraint surface as an attractor.

This technique is also known as a constraint-damping technique. Almost all the current popular formulations used in large-scale numerical simulations include this implementation. The purpose of this article is to find a better way of adjusting the evolution equations to realize $\partial_{t} C^{a} \leq 0$.

\section{B. Idea of $C^{2}$-adjusted systems}

Fiske 24] proposed a way of adjusting the evolution equations which we call " $C^{2}$-adjusted systems";

$$
\partial_{t} u^{i}=f\left(u^{i}, \partial_{j} u^{i}, \cdots\right)-\kappa^{i j}\left(\frac{\delta C^{2}}{\delta u^{j}}\right)
$$

where $\kappa^{i j}$ is a positive-definite constant coefficient and $C^{2}$ is the norm of the constraints, which is defined as $C^{2} \equiv \int C_{a} C^{a} d^{3} x$. The term $\left(\delta C^{2} / \delta u^{j}\right)$ is the functional derivative of $C^{2}$ with respect to $u^{j}$. The associated constraint propagation equation becomes

$$
\partial_{t} C^{2}=h\left(C^{a}, \partial_{i} C^{a}, \cdots\right)-\int d^{3} x\left(\frac{\delta C^{2}}{\delta u^{i}}\right) \kappa^{i j}\left(\frac{\delta C^{2}}{\delta u^{j}}\right) .
$$

The motivation for this adjustment is to naturally obtain the constraint-damping system, $\partial_{t} C^{2}<0$. If we set $\kappa^{i j}$ so that the second term of the right-hand side of (2.7) becomes larger than the first term, then $\partial_{t} C^{2}$ becomes negative, which indicates that constraint violations are expected to decay to zero. Fiske presented numerical examples of the Maxwell system and the linearized ADM and BSSN formulations, and concluded that this method actually reduces constraint violations as expected. In our previous work [26], we applied the $C^{2}$-adjusted system to the (full) ADM formulation and derived the constraint propagation equations. We confirmed that $\partial_{t} C^{2}<0$ is expected in the flat spacetime. We performed numerical tests with the $C^{2}$-adjusted ADM formulation using the Gowdy wave testbed, and confirmed that the violations of the constraint are lower than those of the standard ADM formulation. The simulation continues 1.7 times longer than that of the standard ADM formulation with the magnitude of the violations of the constraint less than order $O\left(10^{0}\right)$. 


\section{APPLICATION TO BSSN FORMULATION}

\section{A. Standard BSSN Formulation}

We work with the widely used notation of the BSSN system. That is, the dynamical variables $\left(\varphi, K, \widetilde{\gamma}_{i j}, \widetilde{A}_{i j}, \widetilde{\Gamma}^{i}\right)$ as the replacement of the variables of the ADM formulation, $\left(\gamma_{i j}, K_{i j}\right)$, where

$$
\begin{aligned}
\varphi & \equiv(1 / 12) \log \left(\operatorname{det}\left(\gamma_{i j}\right)\right), \\
K & \equiv \gamma^{i j} K_{i j}, \\
\widetilde{\gamma}_{i j} & \equiv e^{-4 \varphi} \gamma_{i j}, \\
\widetilde{A}_{i j} & \equiv e^{-4 \varphi}\left(K_{i j}-(1 / 3) \gamma_{i j} K\right), \text { and } \\
\widetilde{\Gamma}^{i} & \equiv \widetilde{\gamma}^{m n} \widetilde{\Gamma}_{m n}^{i} .
\end{aligned}
$$

The BSSN evolution equations are, then,

$$
\begin{aligned}
\partial_{t} \varphi= & -(1 / 6) \alpha K+(1 / 6)\left(\partial_{i} \beta^{i}\right)+\beta^{i}\left(\partial_{i} \varphi\right), \\
\partial_{t} K= & \alpha \widetilde{A}_{i j} \widetilde{A}^{i j}+(1 / 3) \alpha K^{2}-D_{i} D^{i} \alpha+\beta^{i}\left(\partial_{i} K\right) \\
\partial_{t} \widetilde{\gamma}_{i j}= & -2 \alpha \widetilde{A}_{i j}-(2 / 3) \widetilde{\gamma}_{i j}\left(\partial_{\ell} \beta^{\ell}\right) \\
& +\widetilde{\gamma}_{j \ell}\left(\partial_{i} \beta^{\ell}\right)+\widetilde{\gamma}_{i \ell}\left(\partial_{j} \beta^{\ell}\right)+\beta^{\ell}\left(\partial_{\ell} \widetilde{\gamma}_{i j}\right), \\
\partial_{t} \widetilde{A}_{i j}= & \alpha K \widetilde{A}_{i j}-2 \alpha \widetilde{A}_{i \ell} \widetilde{A}_{j}^{\ell}+\alpha e^{-4 \varphi} R_{i j}{ }^{\mathrm{TF}} \\
& -e^{-4 \varphi}\left(D_{i} D_{j} \alpha\right)^{\mathrm{TF}}-(2 / 3) \widetilde{A}_{i j}\left(\partial_{\ell} \beta^{\ell}\right) \\
& +\left(\partial_{i} \beta^{\ell}\right) \widetilde{A}_{j \ell}+\left(\partial_{j} \beta^{\ell}\right) \widetilde{A}_{i \ell}+\beta^{\ell}\left(\partial_{\ell} \widetilde{A}_{i j}\right), \\
\partial_{t} \widetilde{\Gamma}^{i}= & 2 \alpha\left\{6\left(\partial_{j} \varphi\right) \widetilde{A}^{i j}+\widetilde{\Gamma}^{i}{ }_{j \ell} \widetilde{A}^{j \ell}-(2 / 3) \widetilde{\gamma}^{i j}\left(\partial_{j} K\right)\right\} \\
& -2\left(\partial_{j} \alpha\right) \widetilde{A}^{i j}+(2 / 3) \widetilde{\Gamma}^{i}\left(\partial_{j} \beta^{j}\right)+(1 / 3) \widetilde{\gamma}^{i j}\left(\partial_{\ell} \partial_{j} \beta^{\ell}\right) \\
& +\beta^{\ell}\left(\partial_{\ell} \widetilde{\Gamma}^{i}\right)-\widetilde{\Gamma}^{j}\left(\partial_{j} \beta^{i}\right)+\widetilde{\gamma}^{j \ell}\left(\partial_{j} \partial_{\ell} \beta^{i}\right),
\end{aligned}
$$

where ${ }^{\mathrm{TF}}$ denotes the trace-free part. The Ricci tensor in the BSSN system is normally calculated as

$$
R_{i j} \equiv \widetilde{R}_{i j}+R_{i j}^{\varphi}
$$

where

$$
\begin{aligned}
\widetilde{R}_{i j} \equiv & \widetilde{\gamma}_{n(i} \partial_{j)} \widetilde{\Gamma}^{n}+\widetilde{\gamma}^{\ell m}\left(2 \widetilde{\Gamma}^{k}{ }_{\ell(i} \widetilde{\Gamma}_{j) k m}+\widetilde{\Gamma}_{n \ell j} \widetilde{\Gamma}^{n}{ }_{i m}\right) \\
& -(1 / 2) \widetilde{\gamma}^{m \ell} \widetilde{\gamma}_{i j, m \ell}+\widetilde{\Gamma}^{n} \widetilde{\Gamma}_{(i j) n}, \\
R_{i j}^{\varphi} \equiv & -2 \widetilde{D}_{i} \widetilde{D}_{j} \varphi+4\left(\widetilde{D}_{i} \varphi\right)\left(\widetilde{D}_{j} \varphi\right)-2 \widetilde{\gamma}_{i j} \widetilde{D}_{m} \widetilde{D}^{m} \varphi \\
& -4 \widetilde{\gamma}_{i j}\left(\widetilde{D}^{m} \varphi\right)\left(\widetilde{D}_{m} \varphi\right) .
\end{aligned}
$$

The BSSN system has five constraint equations. The "kinematic" constraint equations, which are the Hamiltonian constraint equation and the momentum constraint equations ( $\mathcal{H}$-constraint and $\mathcal{M}$-constraint, hereafter), are expressed in terms of the BSSN basic variables as

$$
\begin{aligned}
\mathcal{H} \equiv & e^{-4 \varphi} \widetilde{R}-8 e^{-4 \varphi}\left(\widetilde{D}_{i} \widetilde{D}^{i} \varphi+\left(\widetilde{D}^{m} \varphi\right)\left(\widetilde{D}_{m} \varphi\right)\right) \\
& +(2 / 3) K^{2}-\widetilde{A}_{i j} \widetilde{A}^{i j}-(2 / 3) \mathcal{A} K \approx 0, \\
\mathcal{M}_{i} \equiv & -(2 / 3) \widetilde{D}_{i} K+6\left(\widetilde{D}_{j} \varphi\right) \widetilde{A}^{j}{ }_{i}+\widetilde{D}_{j} \widetilde{A}^{j}{ }_{i} \\
& -2\left(\widetilde{D}_{i} \varphi\right) \mathcal{A} \approx 0,
\end{aligned}
$$

respectively, where $\widetilde{D}_{i}$ is the covariant derivative associated with $\widetilde{\gamma}_{i j}$ and $\widetilde{R}=\widetilde{\gamma}^{i j} \widetilde{R}_{i j}$. Because of the introduction of new variables, there are additional "algebraic" constraint equations:

$$
\begin{aligned}
\mathcal{G}^{i} & \equiv \widetilde{\Gamma}^{i}-\widetilde{\gamma}^{j \ell} \widetilde{\Gamma}^{i}{ }_{j \ell} \approx 0, \\
\mathcal{A} & \equiv \widetilde{A}^{i j} \widetilde{\gamma}_{i j} \approx 0, \\
\mathcal{S} & \equiv \operatorname{det}\left(\widetilde{\gamma}_{i j}\right)-1 \approx 0,
\end{aligned}
$$

which we call the $\mathcal{G}$-, $\mathcal{A}$-, and $\mathcal{S}$-constraints, respectively, hereafter. If the algebraic constraint equations, (3.16)(3.18), are not satisfied, the BSSN formulation and ADM formulation are not equivalent mathematically.

\section{B. $C^{2}$-adjusted BSSN Formulation}

The $C^{2}$-adjusted BSSN evolution equations are formally written as

$$
\begin{aligned}
& \partial_{t} \varphi=(3.6)-\lambda_{\varphi}\left(\frac{\delta C^{2}}{\delta \varphi}\right), \\
& \partial_{t} K=(3.7)-\lambda_{K}\left(\frac{\delta C^{2}}{\delta K}\right), \\
& \partial_{t} \widetilde{\gamma}_{i j}=(3.8)-\lambda_{\widetilde{\gamma} i j m n}\left(\frac{\delta C^{2}}{\delta \widetilde{\gamma}_{m n}}\right), \\
& \partial_{t} \widetilde{A}_{i j}=(3.9)-\lambda_{\widetilde{A} i j m n}\left(\frac{\delta C^{2}}{\delta \widetilde{A}_{m n}}\right), \\
& \partial_{t} \widetilde{\Gamma}^{i}=(3.10) \\
&-\lambda_{\widetilde{\Gamma}}^{i j}\left(\frac{\delta C^{2}}{\delta \widetilde{\Gamma}^{j}}\right),
\end{aligned}
$$

where all the coefficients $\lambda_{\varphi}, \lambda_{K}, \lambda_{\widetilde{\gamma} i j m n}, \lambda_{\widetilde{A} i j m n}$, and $\lambda_{\widetilde{\Gamma}}^{i j}$ are positive definite. $C^{2}$ is a function of the constraints $\mathcal{H}, \mathcal{M}_{i}, \mathcal{G}^{i}, \mathcal{A}$, and $\mathcal{S}$, which we set as

$$
\begin{aligned}
C^{2}=\int & \left(\mathcal{H}^{2}+\gamma^{i j} \mathcal{M}_{i} \mathcal{M}_{j}+c_{G} \gamma_{i j} \mathcal{G}^{i} \mathcal{G}^{j}\right. \\
& \left.+c_{A} \mathcal{A}^{2}+c_{S} \mathcal{S}^{2}\right) d^{3} x
\end{aligned}
$$

where, $c_{G}, c_{A}$, and $c_{S}$ are Boolean parameters (0 or 1 ). These three parameters are introduced to prove the necessity of the algebraic constraint terms in (3.24).

The adjusted terms in (3.19)-3.23) are then written down explicitly, as shown in Appendix $\mathrm{A}$. The constraint propagation equations of this system are also derived for the Minkowskii background, as shown in Appendix B.

Now we discuss the effect of the algebraic constraints. From (B1)-(B5), we see that the constraints affect each others. The constraint propagation equations of the algebraic constraints, (B3)-(B5), include $c_{G}\left(\lambda_{\widetilde{\gamma}} \Delta \delta^{a}{ }_{b}-\right.$ $\left.2 \lambda_{\widetilde{\Gamma}} \delta^{a}{ }_{b}\right) \mathcal{G}^{b},-6 c_{A} \lambda_{\widetilde{A}} \mathcal{A}$, and $-6 c_{S} \lambda_{\widetilde{\gamma}} \mathcal{S}$, respectively. These terms contribute to reduce the violations of each constraint if $c_{G}, c_{A}$, and $c_{S}$ are non-zero. Therefore, we adopt $c_{G}=c_{A}=c_{S}=1$ in (3.24);

$$
C^{2}=\int\left(\mathcal{H}^{2}+\gamma^{i j} \mathcal{M}_{i} \mathcal{M}_{j}+\gamma_{i j} \mathcal{G}^{i} \mathcal{G}^{j}+\mathcal{A}^{2}+\mathcal{S}^{2}\right) d^{3} x
$$


This discussion is considered only from the viewpoint of the inclusion of the diffusion terms. In order to validate this decision, we perform some numerical examples in Sec:IV]

\section{C. $\widetilde{A}$-adjusted BSSN System}

In 22], two of the authors reported some examples of adjusted systems for the BSSN formulation. The authors investigated the signatures of eigenvalues of the coefficient matrix of the constraint propagation equations, and concluded three of the examples to be the best candidates for the adjustment. The actual numerical tests were performed later [23] using the gauge-wave, linearwave, and polarized Gowdy wave testbeds. The most robust system among the three examples for these three testbeds was the $\widetilde{A}$-adjusted BSSN formulation, which replaces (3.9) in the standard BSSN system with

$$
\partial_{t} \widetilde{A}_{i j}=(\underline{3.9})+\kappa_{A} \alpha \widetilde{D}_{(i} \mathcal{M}_{j)},
$$

where $\kappa_{A}$ is a constant. If $\kappa_{A}$ is set as positive, the violations of the constraints are expected to be damped in flat spacetime [22]. We also use the $\widetilde{A}$-adjusted BSSN system for comparison in the following numerical tests.

\section{NUMERICAL EXAMPLES}

We test the three systems $\left(C^{2}\right.$-adjusted BSSN, $\widetilde{A}$ adjusted BSSN, and standard BSSN) in numerical evolutions using the gauge-wave and polarized Gowdy wave spacetimes, which are the standard tests for comparisons of formulations in numerical relativity, and are known as apples-with-apples testbeds [27]. We also performed the linear-wave testbed but the violations of the constraint are negligible; thus, we employ only the above two testbeds in this article. These tests have been used by several groups and were reported in the same manner (e.g., [23, 28 30]).

For simplicity, we set the coefficient parameters in (3.21)-3.23) to $\lambda_{\widetilde{\gamma} i j m n}=\lambda_{\widetilde{\gamma}} \delta_{i m} \delta_{j n}, \lambda_{\widetilde{A} i j m n}=\lambda_{\widetilde{A}} \delta_{i m} \delta_{j n}$, and $\lambda_{\widetilde{\Gamma}}^{i j}=\lambda_{\widetilde{\Gamma}} \delta^{i j}$ with non-negative coefficient constant parameters $\lambda_{\widetilde{\gamma}}, \lambda_{\widetilde{A}}$, and $\lambda_{\widetilde{\Gamma}}$. Our code passes the convergence test with second-order accuracy. We list the figures in this article in Table \for reader's convenience.

\section{A. Gauge-wave Testbed}

\section{Metric and Parameters}

The metric of the gauge-wave test is

$$
d s^{2}=-H d t^{2}+H d x^{2}+d y^{2}+d z^{2},
$$

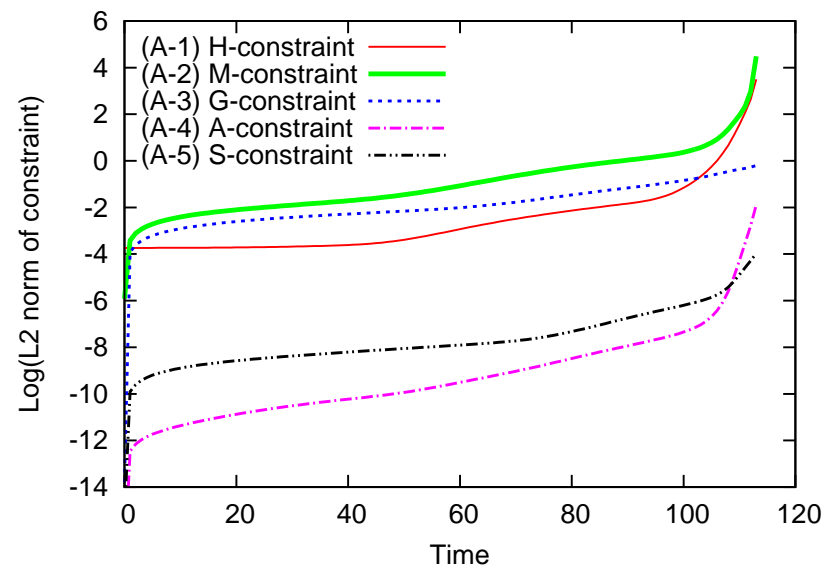

FIG. 1. L2 norm of each constraint violation in the gaugewave evolution using the standard BSSN formulation. The vertical axis is the logarithm of the L2 norm of the constraints and the horizontal axis is time. We see the evolution stops at $t=110$ due to the growth of $\mathcal{M}$-constraint violation.

where

$$
H=1-A \sin (2 \pi(x-t) / d),
$$

which describes a sinusoidal gauge wave of amplitude $A$ propagating along the $x$-axis. The nontrivial extrinsic curvature is

$$
K_{x x}=-\frac{\pi A}{d} \frac{\cos \left(\frac{2 \pi(x-t)}{d}\right)}{\sqrt{1-A \sin \frac{2 \pi(x-t)}{d}}} .
$$

Following [27], we chose the numerical domain and parameters as follows:

- Gauge-wave parameters: $d=1$ and $A=10^{-2}$.

- Simulation domain: $x \in[-0.5,0.5], y=z=0$.

- Grid: $x^{n}=-0.5+(n-1 / 2) d x$ with $n=1, \cdots, 100$, where $d x=1 / 100$.

- Time step: $d t=0.25 d x$.

- Boundary conditions: Periodic boundary condition in $x$-direction and planar symmetry in $y$ - and $z$ directions.

- Gauge conditions:

$$
\partial_{t} \alpha=-\alpha^{2} K, \quad \beta^{i}=0 .
$$

- Scheme: second-order iterative Crank-Nicolson.

\section{Constraint Violations and Their Dampings}

Figure 1 shows the violations of five constraint equations $\mathcal{H}, \mathcal{M}_{i}, \mathcal{G}^{i}, \mathcal{A}$, and $\mathcal{S}$ for the gauge-wave evolution 
TABLE I. List of figures.

\begin{tabular}{|c|c|c|}
\hline & $\begin{array}{l}\text { gauge-wave test } \\
\text { \$VA }\end{array}$ & $\begin{array}{l}\text { Gowdy wave test } \\
\text { gIVB }\end{array}$ \\
\hline $\begin{array}{l}\text { (A) standard BSSN (3.6)-(3.10) } \\
\text { (constraint propagation, see App. C) }\end{array}$ & $\begin{array}{l}\text { Fig.1 norm each } \\
\text { Fig[2 norm all }\end{array}$ & $\begin{array}{l}\text { Fig }[6] \text { norm each } \\
\text { Fig] norm all }\end{array}$ \\
\hline $\begin{array}{l}\text { (B) } \widetilde{A} \text {-adjusted BSSN } \\
(3.6)-(3.8),(3.10) \text {, and }(3.26) \\
\text { (constraint propagation, see App. B })\end{array}$ & $\begin{array}{l}\text { Fig } 2 \text { norm all } \\
\text { Fig } 3 \text { norm each }\end{array}$ & Fig[7norm all \\
\hline $\begin{array}{l}\text { (C) } C^{2} \text {-adjusted BSSN }(3.19)-(3.23) \\
\text { (constraint propagation, see App. B })\end{array}$ & $\begin{array}{l}\text { Fig } 2 \text { norm all } \\
\text { Fig } 3 \text { norm each } \\
\text { Fig } 4 \text { adjusted ratio } \\
\text { Fig } 5 \text { (3.25) test }\end{array}$ & $\begin{array}{l}\text { Fig } 7 \text { norm all } \\
\text { Fig } 8 \text { norm each } \\
\text { Fig } 9 \text { adjusted ratio } \\
\text { Fig } 10 \text { (3.25) test }\end{array}$ \\
\hline
\end{tabular}

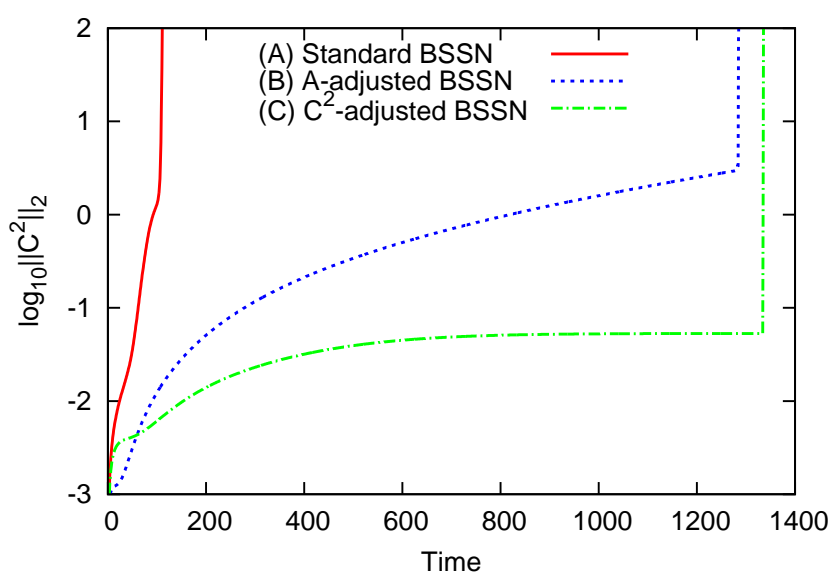

FIG. 2. L2 norm of all the constraints in gauge-wave evolution comparing three BSSN formulations: (A) standard BSSN formulation (solid line), (B) $\widetilde{A}$-adjusted BSSN formulation (dotted line), and (C) $C^{2}$-adjusted BSSN formulation (dot-dashed line). The adopted parameters are $\kappa_{A}=10^{-1.6}$ for $(\mathrm{B})$, and $\lambda_{\varphi}=10^{-8.5}, \lambda_{K}=10^{-8.4}, \lambda_{\widetilde{\gamma}}=10^{-7.3}$, $\lambda_{\widetilde{A}}=10^{-2.5}$, and $\lambda_{\widetilde{\Gamma}}=10^{-1.8}$ for $(\mathrm{C})$ to minimize $C^{2}$ at $t=1000$. The constraint violations of the $\widetilde{A}$-adjusted BSSN formulation, (B), increase with time and the simulation stops before $t=1300$, while those of the $C^{2}$-adjusted BSSN formulation, $(\mathrm{C})$, remain at $O\left(10^{-1}\right)$ until $t=1300$ and the simulation stops at $t=1350$.

using the standard BSSN formulation. The violation of the $\mathcal{M}$-constraint, line (A-2), is the largest during the evolution, while the violations of both the $\mathcal{A}$-constraint and $\mathcal{S}$-constraint are negligible. This is the starting point for improving the BSSN formulation.

Applying the adjustment procedure, the lifetime of the standard BSSN evolution is increased at least 10-fold. In Fig 2, we plot the L2 norm of the constraints, (3.25), of three BSSN evolutions: (A) the standard BSSN formulation (3.6)-(3.10), (B) the $\widetilde{A}$-adjusted BSSN formulation $(3.6)-(3.8),(3.10)$, and (3.26), and (C) the $C^{2}$-adjusted BSSN formulation (3.19)-(3.23). For the standard BSSN case, we see the violation of constraint monotonically increases in the earlier stage, while other two adjusted cases keep it smaller. We can say that the $C^{2}$-adjusted formulation is the most robust one against the violation of constraints between three.

We plot the norm of each constraint equation in Fig 3 , First, we see that the violation of the $\mathcal{M}$-constraint for the two adjusted BSSN formulations [the lines (B-2) and (C-2) in Fig 3 are less than that of the standard BSSN formulation in Fig 1. This behavior would be explained from the constraint propagation equations, where we see the terms $\lambda_{\widetilde{A}} \Delta \mathcal{M}_{a}$ and $(1 / 2) \kappa_{A} \Delta \mathcal{M}_{i}$ in (B2) and (B7), respectively. These terms contribute to reduce the violations of the $\mathcal{M}$-constraint. This is the main consequence of the two adjusted BSSN formulations.

Second, we also find that the violations of the $\mathcal{A}$ constraint and $\mathcal{S}$-constraint are larger than those in Fig 1 . From constraint propagation equations (B4) and (C4), the violation of the $\mathcal{A}$-constraint is triggered by the $\mathcal{M}$ and $\mathcal{A}$-constraints. The increase in the violations of the $\mathcal{A}$-constraint is caused by the term $2 \lambda_{\widetilde{A}} \delta^{i j}\left(\partial_{i} \mathcal{M}_{j}\right)$. Similarly, in (B5) and (C5), the violation of the $\mathcal{S}$-constraint is triggered by only the $\mathcal{A}$-constraint since the magnitude of $\lambda_{\widetilde{\gamma}}$ is negligible. Therefore, the increase in the violation of the $\mathcal{S}$-constraint is due to the violation of the $\mathcal{A}$-constraint.

From (A1) and A3), it can be seen that the adjusted terms of the evolution equations of $\varphi$ and $\widetilde{\gamma}_{i j}$ include second-order derivative terms of the $\mathcal{H}$-constraint. This means that these evolution equations include fourthorder derivative terms of the dynamical variables. In order to investigate the magnitudes of the adjusted terms, we show in Fig, 4 the ratio of the adjusted terms to that of the original terms in each evolution equation. We see that the magnitudes of the adjusted terms of $\varphi$ and $\widetilde{\gamma}_{i j}$ are reasonably small.

In the simulations with the $C^{2}$-adjusted BSSN formulation, the largest violation is the $\mathcal{S}$-constraint. The $\mathcal{S}$ constraint depends only on the dynamical variables $\widetilde{\gamma}_{i j}$, so that there is no other choice than setting $\lambda_{\widetilde{\gamma}}$ for controlling $\mathcal{S}$-constraint, as can be seen from (B5). However, we must set $\lambda_{\widetilde{\gamma}}$ to a value as small as possible since the adjusted term of $\widetilde{\gamma}_{i j}$ includes higher derivatives of $\widetilde{\gamma}_{i j}$. Therefore, it is hard to control the $\mathcal{S}$-constraint, and we have not yet found an appropriate set of parameters. This will remain as a future problem of this $C^{2}$-adjusted 

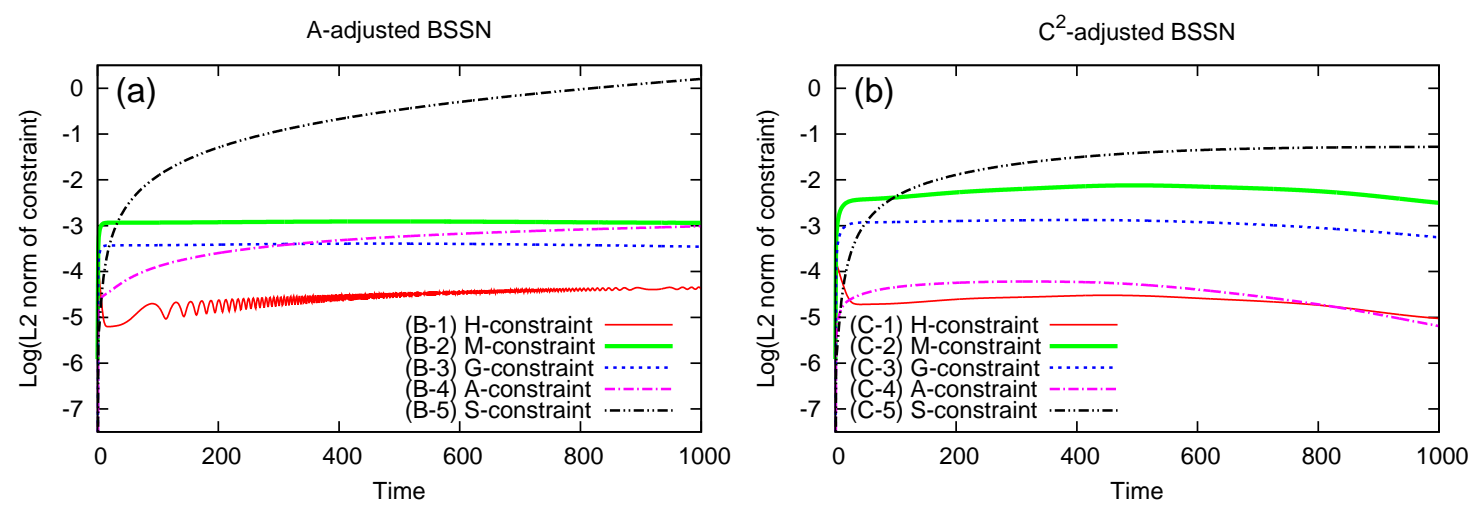

FIG. 3. L2 norm of each constraint in the gauge-wave evolution using the $\widetilde{A}$-adjusted BSSN formulation [panel (a)] and $C^{2}$-adjusted BSSN formulation [panel (b)]. The parameters $\kappa_{A}, \lambda_{\varphi}, \lambda_{K}, \lambda_{\tilde{\gamma}}, \lambda_{\widetilde{A}}$, and $\lambda_{\widetilde{\Gamma}}$ are the same as those in Fig 2] In both panels, we see that the violations of the $\mathcal{H}$-constraint [the lines (B-1) and (C-1)], the $\mathcal{M}$-constraint [(B-2) and (C-2)], and the $\mathcal{G}$-constraint [(B-3) and (C-3)] are less than those for the standard BSSN formulation in Fig 1 However, the violations of the $\mathcal{A}$-constraint [(B-4) and (C-4)] and the $\mathcal{S}$-constraint [(B-5) and (C-5)] are larger. Line (B-5) overlaps with line (B) in Fig 2 after $t=100$, and line (C-5) overlaps with line (C) in Fig 2 after $t=500$.

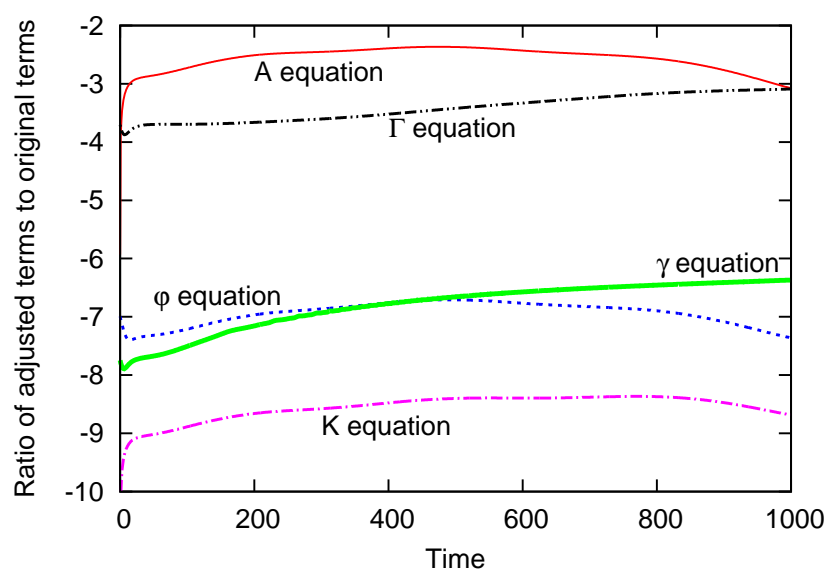

FIG. 4. L2 norm of the ratio (adjusted terms)/(original terms) of each evolution equation of the $C^{2}$-adjusted BSSN formulation, (3.19)-3.23), in the gauge-wave test. We see that the largest ratio is the evolution equation of $\widetilde{A}_{i j}$. The corrections to $\varphi, K$, and $\widetilde{\gamma}_{i j}$ evolution equations are reasonably small.

\section{BSSN system.}

We also investigated the sensitivity of the parameters in the $C^{2}$-adjusted BSSN evolutions. We compared evolutions with setting only one of the parameters, $\left(\lambda_{\varphi}, \lambda_{K}, \lambda_{\widetilde{\gamma}}, \lambda_{\widetilde{A}}, \lambda_{\widetilde{\Gamma}}\right)$, nonzero. Since the key of the damping of the violation of constraints is the $\mathcal{M}$ constraint, and $\left(\lambda_{K}, \lambda_{\widetilde{A}}\right)$ controls the violation of $\mathcal{M}$ constraint directly by (B2), we mention here only the dependence on $\lambda_{K}$ and $\lambda_{\widetilde{A}}$. We found that constraintdamping feature changes sensitively by both $\lambda_{K}$ and $\lambda_{\widetilde{A}}$, among them setting $\lambda_{\widetilde{A}}$ is important to control the $\mathcal{M}$ constraint violation. We see the best controlled evolution

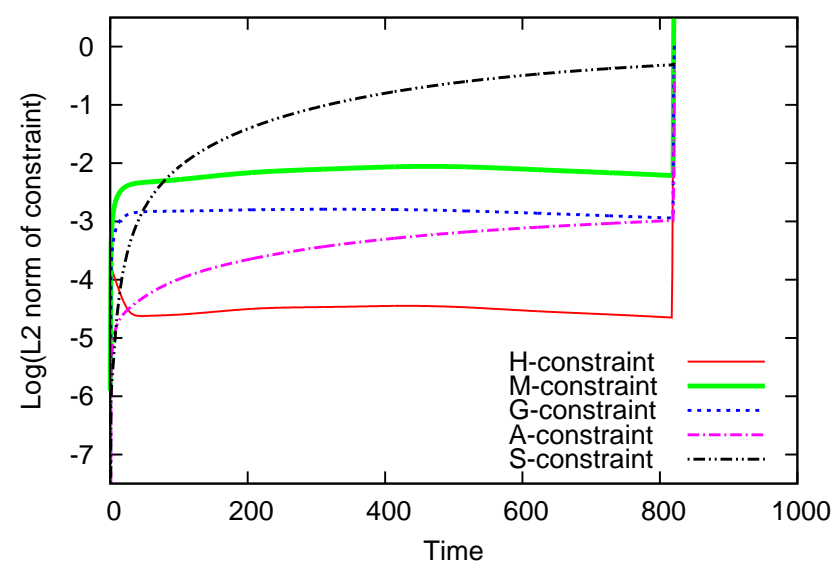

FIG. 5. Difference with the definition of $C^{2}$, 3.25), in the damping of each constraint violation with $c_{G}=c_{A}=c_{S}=0$. The parameters $\lambda_{\varphi}, \lambda_{K}, \lambda_{\widetilde{\gamma}}, \lambda_{\widetilde{A}}$, and $\lambda_{\widetilde{\Gamma}}$ are the same as those in Fig 2 The simulation stops since the violations of the constraints sudden increase at $t=800$.

with $\lambda_{\widetilde{A}}=10^{-3}$, than $10^{-2}$ and $10^{-4}$.

\section{Contribution of Algebraic Constraints in Definition of $C^{2}$}

In Sec IIB we defined $C^{2}$, (3.25), including the algebraic constraints. We check this validity by turning off the algebraic constraints in (3.25). The result is shown in Fig 5 , where we see the simulation stops at $t=800$ due to a sudden increase in the violation of the constraints. This confirms that the algebraic constraints play an important role of damping of the violations of constraints. We also 
tested with other combinations of Boolean parameters $\left(c_{G}, c_{A}, c_{S}\right)$, and confirmed that the best controlled evolution is realized when $c_{G}=c_{A}=c_{S}=1$.

\section{B. Gowdy-wave Testbed}

\section{Metric and Parameters}

The metric of the polarized Gowdy wave is given by

$$
d s^{2}=t^{-1 / 2} e^{\lambda / 2}\left(-d t^{2}+d x^{2}\right)+t\left(e^{P} d y^{2}+e^{-P} d z^{2}\right)
$$

where $P$ and $\lambda$ are functions of $x$ and $t$. The forward direction of the time coordinate $t$ corresponds to the expanding universe, and $t=0$ corresponds to the cosmological singularity.

For simple forms of the solutions, $P$ and $\lambda$ are given by

$$
\begin{aligned}
P= & J_{0}(2 \pi t) \cos (2 \pi x), \\
\lambda= & -2 \pi t J_{0}(2 \pi t) J_{1}(2 \pi t) \cos ^{2}(2 \pi x)+2 \pi^{2} t^{2}\left[J_{0}^{2}(2 \pi t)\right. \\
& \left.+J_{1}^{2}(2 \pi t)\right]-(1 / 2)\left\{(2 \pi)^{2}\left[J_{0}^{2}(2 \pi)+J_{1}^{2}(2 \pi)\right]\right. \\
& \left.-2 \pi J_{0}(2 \pi) J_{1}(2 \pi)\right\},
\end{aligned}
$$

where $J_{n}$ is the Bessel function.

Following [27], a new time coordinate $\tau$, which satisfies harmonic slicing, is obtained by the coordinate transformation

$$
t(\tau)=k e^{c \tau}
$$

where $k$ and $c$ are arbitrary constants. We also follow [27] by setting $k, c$, and the initial time $t_{0}$ as

$$
\begin{aligned}
k & \sim 9.67076981276405, \quad c \sim 0.002119511921460 \\
t_{0} & =9.87532058290982,
\end{aligned}
$$

so that the lapse function in the new time coordinate is unity and $t=\tau$ at the initial time.

We also use the following parameters specified in [27].

- Simulation domain: $x \in[-0.5,0.5], y=z=0$.

- Grid: $x_{n}=-0.5+(n-(1 / 2)) d x, n=1, \cdots, 100$, where $d x=1 / 100$.

- Time step: $d t=0.25 d x$.

- Boundary conditions: Periodic boundary condition in $x$-direction and planar symmetry in $y$ - and $z$ directions.

- Gauge conditions: $\partial_{t} \alpha=-\alpha^{2} K, \beta^{i}=0$.

- Scheme: second-order iterative Crank-Nicolson.

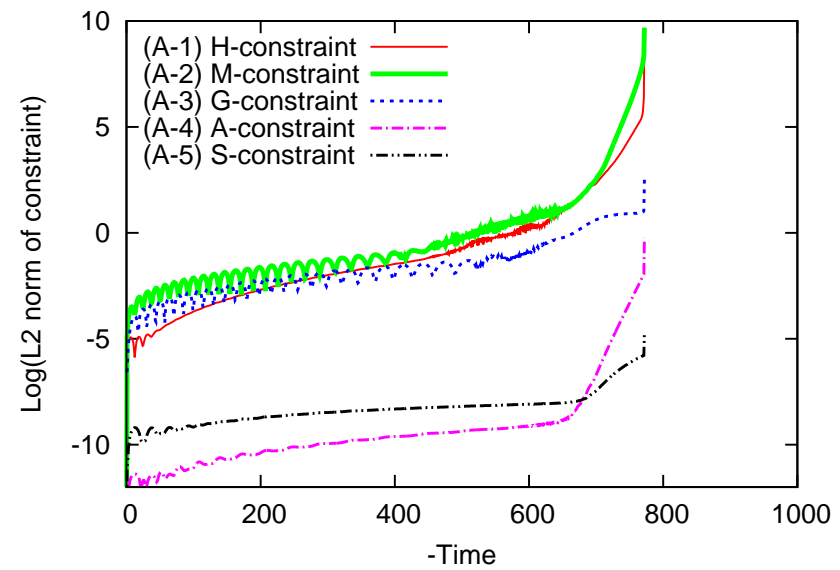

FIG. 6. L2 norm of each constraint equation in the polarized Gowdy wave evolution using the standard BSSN formulation. The vertical axis is the logarithm of the L2 norm of the constraint and the horizontal axis is backward time.

\section{Constraint Violations and Their Dampings}

We begin showing the case of the standard BSSN formulation, (3.6)-3.10). Figure 6 shows the L2 norm of the violations of the constraints as a function of backward time $(-t)$. We see that the violation of the $\mathcal{M}$ constraint is the largest at all times and that all the violations of constraints increase monotonically with time. [Comparing with the result in [23], our code shows that the $\mathcal{H}$-constraint $(\mathrm{A}-1)$ remains at the same level but the $\mathcal{M}$-constraint (A-2) is smaller.]

Similar to the gauge-wave test, we compare the violations of $C^{2}$ for three types of BSSNs in Fig.7. In the case of the $\widetilde{A}$-adjusted BSSN formulation, the violation of the constraints increases if we set $\left|\kappa_{A}\right|$ larger than $10^{-0.2}$. In the case of the $C^{2}$-adjusted BSSN formulation, it increases if we set $\left|\lambda_{\widetilde{A}}\right|$ larger than $10^{-1.2}$. Note that the signatures of the above $\kappa_{A}$ and $\lambda_{\mathrm{s}}$ are negative, contrary to the predictions in [22] and Sec III] respectively. This is because these simulations are performed with backward time.

As shown in Fig.7, the violations of $C^{2}$ for the standard BSSN formulation and the $\widetilde{A}$-adjusted BSSN formulation increase monotonically with time, while that for the $C^{2}$ adjusted BSSN formulation decreases after $t=-200$. To investigate the reason of this rapid decay after $t=$ -200 , we plot each constraint violation in Fig 8. We see that the violations of the $\mathcal{A}$-constraint and $\mathcal{S}$-constraint increase with negative time, in contrast to the standard BSSN formulation, and those of the $\mathcal{M}$-constraint and $\mathcal{G}$-constraint decrease after $t=-200$. The propagation equation of the $\mathcal{M}$-constraint, (B2), includes the term $-2 c_{A} \lambda_{\widetilde{A}} \partial_{a} \mathcal{A}$, which contributes to constraint damping. Similarly, the propagation equation of the $\mathcal{G}$-constraint, (B3), includes $\delta^{a b}\left\{(1 / 2) \lambda_{\widetilde{\gamma}} \partial_{b} \Delta+2 \lambda_{\widetilde{\Gamma}} \partial_{b}\right\} \mathcal{H}-c_{S} \lambda_{\widetilde{\gamma}} \delta^{a b} \partial_{b} \mathcal{S}$; the decay of the violations of the $\mathcal{G}$-constraint is caused 


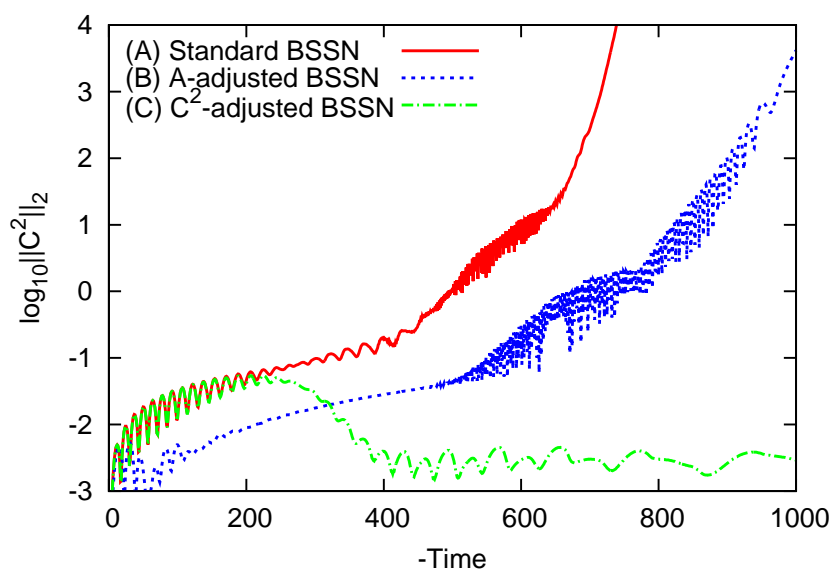

FIG. 7. L2 norm of the constraints, $C^{2}$, of the polarized Gowdy wave tests for the standard BSSN and two adjusted formulations. The vertical axis is the logarithm of the L2 norm of $C^{2}$ and the horizontal axis is backward time. The solid line (A) is the standard BSSN formulation, the dotted line $(\mathrm{B})$ is the $\widetilde{A}$-adjusted BSSN formulation with $\kappa_{A}=$ $-10^{-0.2}$, and the dot-dashed line $(\mathrm{C})$ is the $C^{2}$-adjusted BSSN formulation with $\lambda_{\varphi}=-10^{-10}, \lambda_{K}=-10^{-4.6}, \lambda_{\widetilde{\gamma}}=-10^{-11}$, $\lambda_{\widetilde{A}}=-10^{-1.2}$, and $\lambda_{\widetilde{\Gamma}}=-10^{-14.3}$. Note that the signatures of $\kappa_{A}$ and $\lambda_{\mathrm{s}}$ are negative since the simulations evolve backward. We see that lines (A) and (C) are identical until $t=-200$. Line (C) then decreases and maintains its magnitude under $O\left(10^{-2}\right)$ after $t=-400$. We confirm this behavior until $t=-1500$.

by these terms. Therefore, these terms are considered to become significant of approximately $t=-200$ when the violations of the $\mathcal{A}, \mathcal{H}$, and $\mathcal{S}$-constraints become a certain order of magnitude.

In contrast to the gauge-wave testbed (Fig 4), we prepared Fig 9. which shows the magnitudes of the ratio of the adjusted terms to the original terms. Since the magnitudes of the adjusted terms of $\varphi$ and $\widetilde{\gamma}_{i j}$ can be disregarded, the effect of the reduction of the adjusted terms of $\varphi$ and $\widetilde{\gamma}_{i j}$ is negligible. Therefore, the $C^{2}$-adjusted BSSN evolution in the Gowdy wave can be regarded as maintaining its original hyperbolicity.

We repeated the parameter-dependency survey of $\left(\lambda_{\varphi}, \lambda_{K}, \lambda_{\widetilde{\gamma}}, \lambda_{\widetilde{A}}, \lambda_{\widetilde{\Gamma}}\right)$ for this spacetime evolution. Similar to SecIVA2 , we found that constraint-damping feature is sensitive to both $\lambda_{K}$ and $\lambda_{\widetilde{A}}$, of which $\lambda_{\widetilde{A}}$ works effectively than $\lambda_{K}$. We see the most controlled evolution when $\lambda_{\widetilde{A}}=10^{-1}$, than that of $\lambda_{\widetilde{A}}=10^{0}$ or $\lambda_{\widetilde{A}}=10^{-2}$.

\section{Contribution of Algebraic Constraints in Definition of $C^{2}$}

In Sec IIIB, we investigated the effect of the definition of $C^{2}$. Similar to the gauge-wave tests in the previous subsection, we show the effect of constraint damping caused by the algebraic constraints. In Fig 10, we plot

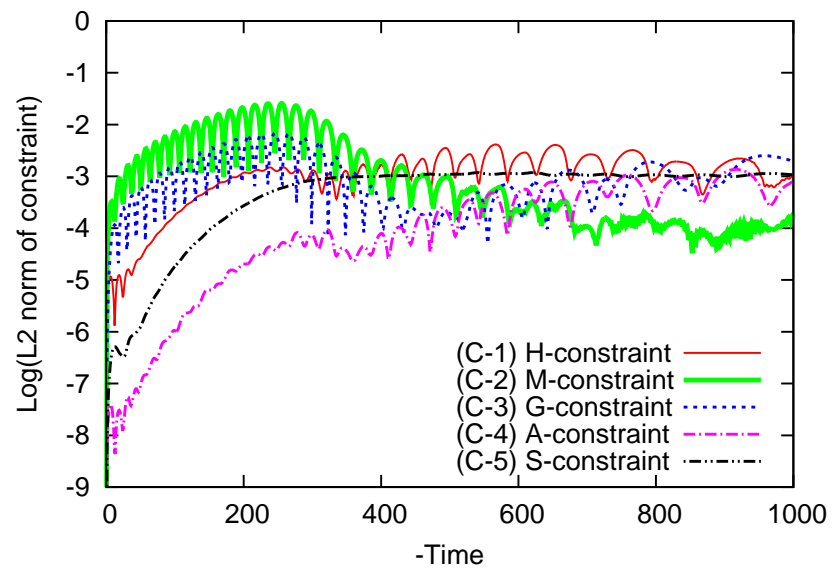

FIG. 8. The same with Fig 6 but for the $C^{2}$-adjusted BSSN formulation. The parameters, $\left(\lambda_{\varphi}, \lambda_{K}, \lambda_{\widetilde{\gamma}}, \lambda_{\widetilde{A}}, \lambda_{\widetilde{\Gamma}}\right)$, are the same with those for (C) in Fig[7 We see that the violation of the $\mathcal{M}$-constraint decreases and becomes the lowest after $t=-700$.

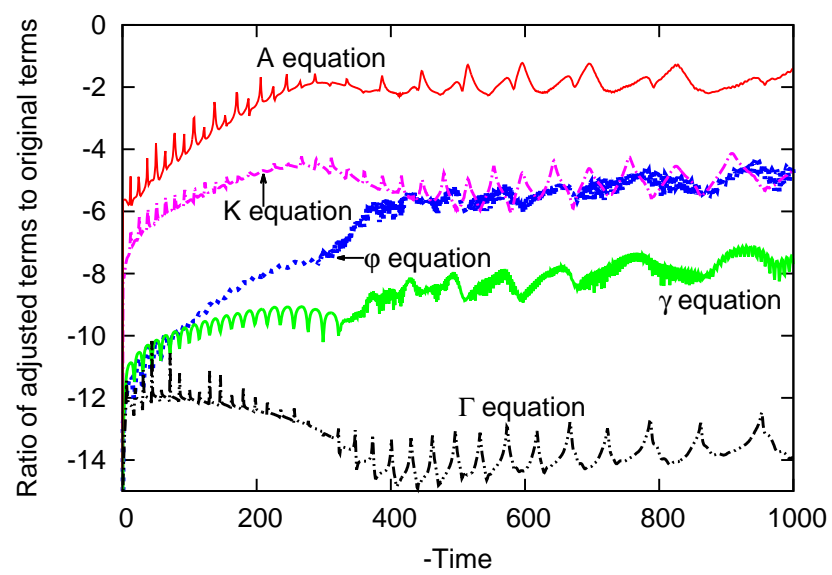

FIG. 9. L2 norm of the ratio (adjusted terms)/(original terms) of each evolution equation for the $C^{2}$-adjusted BSSN formulation, (3.19)- We see that the largest ratio is that for the evolution of $\widetilde{A}_{i j}$. The corrections to the $\widetilde{\gamma}_{i j}$ and $\widetilde{\Gamma}^{i}$ evolution equations are reasonably small.

the violations of all the constraint with $c_{G}=c_{A}=c_{S}=$ 0 . We see that all the violations of the constraints are larger than those in Fig 8 This result is consistent with the discussion in Sec

\section{SUMMARY AND DISCUSSION}

To obtain an evolution system robust against the violation of constraints, we derived a new set of adjusted BSSN equations applying the idea proposed by Fiske 24 which we call a " $C^{2}$-adjusted system." That is, we added 


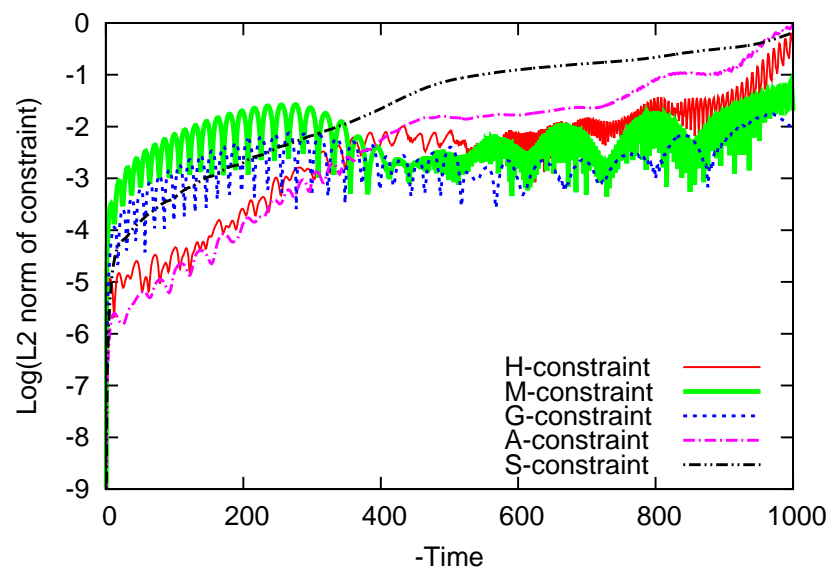

FIG. 10. Difference with the definition of $C^{2}$ with $c_{G}=$ $c_{A}=c_{S}=0$. The coefficient parameters, $\lambda_{\varphi}, \lambda_{K}, \lambda_{\widetilde{\gamma}}, \lambda_{\widetilde{A}}$ and $\lambda_{\widetilde{\Gamma}}$, are all the same as those for (C) in Fig 7 . In comparison with Fig 8 , all the violations of the constraints are larger.

the functional derivatives of the norm of the constraints, $C^{2}$, to the evolution equations [3.19)-(3.23)]. We performed numerical tests in the gauge-wave and Gowdy wave spacetimes and confirmed that the violations of constraints decrease as expected, and that longer and accurate simulation than that of the standard BSSN evolution is available.

The construction of the $C^{2}$-adjusted system is straightforward. However, in BSSN, there are two kinetic constraints and three additional algebraic constraints compared to the ADM system; thus, the definition of $C^{2}$ is a matter of concern. By analyzing constraint propagation equations, we concluded that $C^{2}$ should include all the constraints. This was also confirmed by numerical tests. The importance of such algebraic constraints suggests the similar treatment when we apply this idea to other formulations of the Einstein equation.

To evaluate the reduction of the violations of the constraints, we also compared evolutions with the $\widetilde{A}$ adjusted BSSN formulation proposed in [22]. We concluded that the $C^{2}$-adjusted BSSN formulation exhibits superior constraint damping to both the standard and $\widetilde{A}$-adjusted BSSN formulations. In particular, the lifetimes of the simulations of the $C^{2}$-adjusted BSSN formulation in the gauge-wave and Gowdy wave testbeds are as ten-times and twice as longer than those of the standard BSSN formulation, respectively.

So far, many trials have been reported to improve BSSN formulation (e.g. 22, 31]). Recently, for example, a conformal-traceless Z4 formulation was proposed with its test demonstrations [17]. Among them, Fig.1 of [17] can be compared with our Fig 3 [(B-1) and (C-1)] as the same gauge-wave test. The violation of $\mathcal{H}$-constraint in
$C^{2}$-adjusted evolution looks smaller than that of new Z4 evolution, but regarding the blow-up time of simulations, new Z4 system has advantage.

Fiske reported the applications of the idea of $C^{2}$ adjustment to "linearized" ADM and BSSN formulations in his dissertation [25]. (As he mentioned, his BSSN is not derived from the standard BSSN equations but from a linearized ADM using a new variable, $\Gamma$. His set of BSSN equations also does not include the $\mathcal{A}$ - and $\mathcal{S}$ constraints in our notation.). He observed damping of the constraint violation of five orders of magnitude and the equivalent solution errors in his numerical evolution tests. Our studies show that the full BSSN set of equations with fully adjusted terms also produces the desired constraint-damping results (Fig 2 and Fig 7), although apparent improvements are at fewer orders of magnitude.

When applied this idea to the ADM system [26], we found that the adjustment to the $K_{i j}$-evolution equation is essential. In the present study, we found that the adjustment to the $\widetilde{A}_{i j}$-evolution equation is essential for controlling the constraints. In both cases, the associated adjustment parameters (Lagrangian multipliers), $\lambda_{\widetilde{A}}$ in this study, are sensitive and require fine-tuning. In future, automatic controlling system such that monitoring the order of constraint violations and maintaining them by tuning the parameters automatically would be helpful. Applications of control theory in this direction are being investigated.

The correction terms of the $C^{2}$-adjusted system include higher-order derivatives and are not quasi-linear; thus, little is known mathematically about such systems. These additional terms might effectively act as artificial viscosity terms in fluid simulations, but might also enhance the violation of errors. To investigate this direction further, the next step is to apply the idea to a system in which constraints do not include second-order derivatives of dynamical variables. We are working on the KidderScheel-Teukolsky formulation [10] as an example of such a system, which we will report in the near future.

\section{ACKNOWLEDGMENTS}

This work was partially supported by Grant-in-Aid for Scientific Research Fund of Japan Society of the Promotion of Science No. 22540293 (HS). Numerical computations were carried out on an Altix 3700 BX2 supercomputer at YITP in Kyoto University and on the RIKEN Integrated Cluster of Clusters (RICC).

\section{Appendix A: Additional $C^{2}$-adjusted Terms}

The adjusted terms $\delta C^{2} / \delta \varphi, \delta C^{2} / \delta K, \delta C^{2} / \delta \widetilde{\gamma}_{m n}$, $\delta C^{2} / \delta \widetilde{A}_{m n}$, and $\delta C^{2} / \delta \widetilde{\Gamma}^{a}$ in (3.19)-(3.23) are written as follows: 


$$
\begin{aligned}
& \frac{\delta C^{2}}{\delta \varphi}=2 \bar{H}_{1} \mathcal{H}-2\left(\partial_{a} \bar{H}_{2}^{a}\right) \mathcal{H}-2 \bar{H}_{2}^{a} \partial_{a} \mathcal{H}+2\left(\partial_{a} \partial_{b} \bar{H}_{3}^{a b}\right) \mathcal{H}+2\left(\partial_{a} \bar{H}_{3}^{a b}\right) \partial_{b} \mathcal{H}+2\left(\partial_{b} \bar{H}_{3}^{a b}\right) \partial_{a} \mathcal{H}+2 \bar{H}_{3}^{a b} \partial_{a} \partial_{b} \mathcal{H} \\
& -2\left(\partial_{a} \bar{M}_{1 i}{ }^{a}\right) e^{-4 \varphi} \widetilde{\gamma}^{i j} \mathcal{M}_{j}+8 \bar{M}_{1 i}{ }^{a} e^{-4 \varphi}\left(\partial_{a} \varphi\right) \widetilde{\gamma}^{i j} \mathcal{M}_{j}-2 \bar{M}_{1 i}{ }^{a} e^{-4 \varphi}\left(\partial_{a} \widetilde{\gamma}^{i j}\right) \mathcal{M}_{j}-2 \bar{M}_{1 i}{ }^{a} e^{-4 \varphi} \widetilde{\gamma}^{i j} \partial_{a} \mathcal{M}_{j} \\
& -4 \widetilde{\gamma}^{i j} e^{-4 \varphi} \mathcal{M}_{i} \mathcal{M}_{j}+4 c_{G} e^{4 \varphi} \widetilde{\gamma}_{i j} \mathcal{G}^{i} \mathcal{G}^{j}, \\
& \frac{\delta C^{2}}{\delta K}=2 \bar{H}_{4} \mathcal{H}-2\left(\partial_{\ell} \bar{M}_{2 i}{ }^{\ell}\right) e^{-4 \varphi} \widetilde{\gamma}^{i j} \mathcal{M}_{j}+8 \bar{M}_{2 i}{ }^{\ell} e^{-4 \varphi}\left(\partial_{\ell} \varphi\right) \widetilde{\gamma}^{i j} \mathcal{M}_{j}-2 \bar{M}_{2 i}{ }^{\ell} e^{-4 \varphi}\left(\partial_{\ell} \widetilde{\gamma}^{i j}\right) \mathcal{M}_{j}-2 \bar{M}_{2 i}{ }^{\ell} e^{-4 \varphi} \widetilde{\gamma}^{i j} \partial_{\ell} \mathcal{M}_{j} \text {, } \\
& \frac{\delta C^{2}}{\delta \widetilde{\gamma}_{m n}}=2 \bar{H}_{5}^{m n} \mathcal{H}-2\left(\partial_{i} \bar{H}_{6}^{i m n}\right) \mathcal{H}-2 \bar{H}_{6}^{i m n} \partial_{i} \mathcal{H}+2\left(\partial_{i} \partial_{j} \bar{H}_{7}^{i j m n}\right) \mathcal{H}+2\left(\partial_{i} \bar{H}_{7}^{i j m n}\right) \partial_{j} \mathcal{H}+2\left(\partial_{j} \bar{H}_{7}^{i j m n}\right) \partial_{i} \mathcal{H} \\
& +2 \bar{H}_{7}^{i j m n} \partial_{i} \partial_{j} \mathcal{H}+2 \bar{M}_{3 i}{ }^{m n} e^{-4 \varphi} \widetilde{\gamma}^{i j} \mathcal{M}_{j}-2\left(\partial_{c} \bar{M}_{4 i}{ }^{c m n}\right) e^{-4 \varphi} \widetilde{\gamma}^{i j} \mathcal{M}_{j}+8 \bar{M}_{4 i}{ }^{c m n} e^{-4 \varphi}\left(\partial_{c} \varphi\right) \widetilde{\gamma}^{i j} \mathcal{M}_{j} \\
& -2 \bar{M}_{4 i}{ }^{c m n} e^{-4 \varphi}\left(\partial_{c} \widetilde{\gamma}^{i j}\right) \mathcal{M}_{j}-2 \bar{M}_{4 i}{ }^{c m n} e^{-4 \varphi} \widetilde{\gamma}^{i j} \partial_{c} \mathcal{M}_{j}-e^{-4 \varphi} \widetilde{\gamma}^{i m} \widetilde{\gamma}^{j n} \mathcal{M}_{i} \mathcal{M}_{j}+2 c_{G} G_{1}^{i m n} e^{4 \varphi} \widetilde{\gamma}_{i j} \mathcal{G}^{j} \\
& -2 c_{G}\left(\partial_{\ell} G_{2}^{i m n \ell}\right) e^{4 \varphi} \widetilde{\gamma}_{i j} \mathcal{G}^{j}-8 c_{G} G_{2}^{i m n \ell} e^{4 \varphi}\left(\partial_{\ell} \varphi\right) \widetilde{\gamma}_{i j} \mathcal{G}^{j}-2 c_{G} G_{2}^{i m n \ell} e^{4 \varphi}\left(\partial_{\ell} \widetilde{\gamma}_{i j}\right) \mathcal{G}^{j}-2 c_{G} G_{2}^{i m n \ell} e^{4 \varphi} \widetilde{\gamma}_{i j} \partial_{\ell} \mathcal{G}^{j} \\
& +c_{G} e^{4 \varphi} \mathcal{G}^{m} \mathcal{G}^{n}+2 c_{A} A_{1}^{m n} \mathcal{A}+2 c_{S} S_{1}^{m n} \mathcal{S}, \\
& \frac{\delta C^{2}}{\delta \widetilde{A}_{m n}}=2 \bar{H}_{8}^{m n} \mathcal{H}+2 e^{-4 \varphi} \widetilde{\gamma}^{i j} \bar{M}_{5 i}{ }^{m n} \mathcal{M}_{j}-2\left(\partial_{c} \bar{M}_{6 i}{ }^{c m n}\right) e^{-4 \varphi} \widetilde{\gamma}^{i j} \mathcal{M}_{j}+8 \bar{M}_{6 i}{ }^{c m n} e^{-4 \varphi}\left(\partial_{c} \varphi\right) \widetilde{\gamma}^{i j} \mathcal{M}_{j} \\
& -2 \bar{M}_{6 i}{ }^{c m n} e^{-4 \varphi}\left(\partial_{c} \widetilde{\gamma}^{i j}\right) \mathcal{M}_{j}-2 \bar{M}_{6 i}{ }^{c m n} e^{-4 \varphi} \widetilde{\gamma}^{i j} \partial_{c} \mathcal{M}_{j}+2 c_{A} A_{2}^{m n} \mathcal{A}, \\
& \frac{\delta C^{2}}{\delta \widetilde{\Gamma}^{a}}=2 \bar{H}_{9 a} \mathcal{H}-2\left(\partial_{b} \bar{H}_{10 a}^{b}\right) \mathcal{H}-2 \bar{H}_{10 a}^{b} \partial_{b} \mathcal{H}+2 c_{G} G_{3 a}^{i} e^{4 \varphi} \widetilde{\gamma}_{i j} \mathcal{G}^{j}
\end{aligned}
$$

where

$$
\bar{H}_{8}^{m n}=-2 \widetilde{A}^{m n}-(2 / 3) \widetilde{\gamma}^{m n} K,
$$

$$
\begin{gathered}
\bar{H}_{1}=-4 e^{-4 \varphi} \widetilde{R}+32 e^{-4 \varphi}\left\{\widetilde{D}^{i} \widetilde{D}_{i} \varphi+\left(\widetilde{D}_{i} \varphi\right)\left(\widetilde{D}^{i} \varphi\right)\right\} \\
\bar{H}_{2}^{a}=8 e^{-4 \varphi}\left(\widetilde{\gamma}^{i j} \widetilde{\Gamma}^{a}{ }_{i j}-2 \widetilde{D}^{a} \varphi\right) \\
\bar{H}_{3}^{a b}=-8 e^{-4 \varphi} \widetilde{\gamma}^{a b} \\
\bar{H}_{4}=(4 / 3) K-(2 / 3) \widetilde{\gamma}^{i j} \widetilde{A}_{i j}
\end{gathered}
$$

$$
\begin{aligned}
\bar{H}_{5}^{m n}= & -e^{-4 \varphi} \widetilde{R}^{m n}+e^{-4 \varphi}\left(\partial_{j} \widetilde{\Gamma}^{(m}\right) \widetilde{\gamma}^{n) j} \\
& -2 e^{-4 \varphi} \widetilde{\Gamma}^{k m}{ }_{j} \widetilde{\Gamma}^{j n}{ }_{k}-2 e^{-4 \varphi} \widetilde{\Gamma}^{i \ell(m} \widetilde{\Gamma}^{n)}{ }_{\ell i} \\
& -e^{-4 \varphi} \widetilde{\Gamma}^{a m i} \widetilde{\Gamma}_{a i}{ }^{n}-e^{-4 \varphi} \widetilde{\Gamma}^{m i \ell} \widetilde{\Gamma}^{n}{ }_{\ell i} \\
& +(1 / 2) e^{-4 \varphi} \widetilde{\gamma}_{i j, a \ell} \widetilde{\gamma}^{i j} \widetilde{\gamma}^{a m} \widetilde{\gamma}^{\ell n}+8 e^{-4 \varphi} \widetilde{D}^{m} \widetilde{D}^{n} \varphi \\
& -8 e^{-4 \varphi}\left(\widetilde{D}^{(m} \varphi\right) \widetilde{\Gamma}^{n)}{ }_{i j} \widetilde{\gamma}^{i j}+8 e^{-4 \varphi}\left(\widetilde{D}^{m} \varphi\right)\left(\widetilde{D}^{n} \varphi\right) \\
& +2 \widetilde{A}^{m b} \widetilde{A}^{n}{ }_{b}+(2 / 3) \widetilde{A}^{m n} K, \\
\bar{H}_{6}^{\ell m n}= & e^{-4 \varphi}\left\{\widetilde{\Gamma}^{\ell m n}+2 \widetilde{\Gamma}^{(n m) \ell}+(1 / 2) \Gamma^{\ell} \widetilde{\gamma}^{m n}\right. \\
& \left.+8 \widetilde{\gamma}^{\ell(m}\left(\widetilde{D}^{n)} \varphi\right)-4 \widetilde{\gamma}^{m n} \widetilde{D}^{\ell} \varphi\right\}, \quad(\mathrm{A} 11) \\
& \bar{H}_{7}^{i j m n}=-(1 / 2) e^{-4 \varphi} \widetilde{\gamma}^{m n} \widetilde{\gamma}^{i j}, \quad(\mathrm{~A} 12)
\end{aligned}
$$$$
\bar{H}_{9 a}=(1 / 2) e^{-4 \varphi} \widetilde{\gamma}^{i j} \widetilde{\gamma}_{i j, a},
$$$$
\bar{H}_{10 a}^{b}=e^{-4 \varphi} \delta^{b}{ }_{a},
$$$$
\bar{M}_{1 i}{ }^{a}=6 \widetilde{A}^{a}{ }_{i}-2 \widetilde{A}_{m n} \widetilde{\gamma}^{m n} \delta^{a}{ }_{i},
$$$$
\bar{M}_{2 i}{ }^{j}=-(2 / 3) \delta^{j}{ }_{i},
$$

$$
\begin{aligned}
\bar{M}_{3 i}{ }^{m n}= & -6\left(\widetilde{D}^{(m} \varphi\right) \widetilde{A}^{n)}{ }_{i}+2\left(\widetilde{D}_{i} \varphi\right) \widetilde{A}^{m n}-\widetilde{D}^{(m} \widetilde{A}^{n)}{ }_{i} \\
& +\widetilde{A}^{a(n} \widetilde{\Gamma}^{m)}{ }_{a i}+\widetilde{A}_{i}{ }^{(m} \widetilde{\Gamma}^{n)}{ }_{j \ell} \widetilde{\gamma}^{j \ell},
\end{aligned}
$$

$$
\bar{M}_{4 i}{ }^{c m n}=-\widetilde{\gamma}^{c(n} \widetilde{A}^{m)}{ }_{i}+(1 / 2) \widetilde{\gamma}^{m n} \widetilde{A}^{c}{ }_{i}-(1 / 2) \widetilde{A}^{n m} \delta^{c}{ }_{i},
$$

$$
\begin{aligned}
& \bar{M}_{5 i}{ }^{m n}=6\left(\widetilde{D}^{(m} \varphi\right) \delta^{n)}{ }_{i}-2\left(\widetilde{D}_{i} \varphi\right) \widetilde{\gamma}^{m n}-\delta_{i}{ }^{(m} \widetilde{\Gamma}^{n)}{ }_{j \ell} \widetilde{\gamma}^{j \ell} \\
& +(1 / 2) \widetilde{\gamma}^{m n}{ }_{, i}, \\
& \bar{M}_{6 i}{ }^{c m n}=\widetilde{\gamma}^{c(m} \delta^{n)}{ }_{i}, \\
& G_{1}^{i a b}=\widetilde{\Gamma}^{i a b}+\widetilde{\gamma}^{i(b} \widetilde{\Gamma}_{m n}^{a)} \widetilde{\gamma}^{m n},
\end{aligned}
$$




$$
\begin{gathered}
G_{2}^{i a b \ell}=-\widetilde{\gamma}^{\ell(b} \widetilde{\gamma}^{a) i}+(1 / 2) \widetilde{\gamma}^{a b} \widetilde{\gamma}^{i \ell} \\
G_{3 j}^{i}=\delta^{i}{ }_{j} \\
A_{1}^{a b}=-\widetilde{A}^{a b} \\
A_{2}^{a b}=\widetilde{\gamma}^{a b}
\end{gathered}
$$

$$
S_{1}^{a b}=(1 / 2) \varepsilon^{a j k} \varepsilon^{b n \ell} \widetilde{\gamma}_{j n} \widetilde{\gamma}_{k \ell}
$$

\section{Appendix B: Constraint Propagation Equations of Adjusted BSSN Formulations}

Here we give the constraint propagation equations for the $C^{2}$-adjusted BSSN formulation and the $\widetilde{A}$-adjusted BSSN formulation in Minkowskii spacetime. For simplicity, we set $\lambda_{\widetilde{\gamma} i j m n}=\lambda_{\widetilde{\gamma}} \delta_{i m} \delta_{j n}, \lambda_{\widetilde{A} i j m n}=\lambda_{\widetilde{A}} \delta_{i m} \delta_{j n}$, and $\lambda_{\widetilde{\Gamma}}^{i j}=\lambda_{\widetilde{\Gamma}} \delta^{i j}$. The constraint propagation equations of the $C^{2}$-adjusted BSSN formulation are

$$
\begin{aligned}
\partial_{t} \mathcal{H} & =[\text { Original Terms }]+\left(-128 \lambda_{\varphi} \Delta^{2}-(3 / 2) \lambda_{\widetilde{\gamma}} \Delta^{2}+2 \lambda_{\widetilde{\Gamma}} \Delta\right) \mathcal{H}+c_{G}\left(-(1 / 2) \lambda_{\widetilde{\gamma}} \Delta \partial_{m}-2 \lambda_{\widetilde{\Gamma}} \partial_{m}\right) \mathcal{G}^{m}+3 c_{S} \lambda_{\widetilde{\gamma}} \Delta \mathcal{S}, \\
\partial_{t} \mathcal{M}_{a} & =[\text { Original Terms }]+\left\{(8 / 9) \lambda_{K} \delta^{b c} \partial_{a} \partial_{b}+\lambda_{\widetilde{A}} \Delta \delta_{a}{ }^{c}+\lambda_{\widetilde{A}} \delta^{b c} \partial_{a} \partial_{b}\right\} \mathcal{M}_{c}-2 c_{A} \lambda_{\widetilde{A}} \partial_{a} \mathcal{A}, \\
\partial_{t} \mathcal{G}^{a} & =[\text { Original Terms }]+\delta^{a b}\left((1 / 2) \lambda_{\widetilde{\gamma}} \partial_{b} \Delta+2 \lambda_{\widetilde{\Gamma}} \partial_{b}\right) \mathcal{H}+c_{G}\left(\lambda_{\widetilde{\gamma}} \Delta \delta^{a}{ }_{b}+(1 / 2) \lambda_{\widetilde{\gamma}} \delta^{a c} \partial_{c} \partial_{b}-2 \lambda_{\widetilde{\Gamma}} \delta^{a}{ }_{b}\right) \mathcal{G}^{b}-c_{S} \lambda_{\widetilde{\gamma}} \delta^{a b} \partial_{b} \mathcal{S}, \\
\partial_{t} \mathcal{A} & =[\text { Original Terms }]+2 \lambda_{\widetilde{A}} \delta^{i j}\left(\partial_{i} \mathcal{M}_{j}\right)-6 c_{A} \lambda_{\widetilde{A}} \mathcal{A}, \\
\partial_{t} \mathcal{S} & =[\text { Original Terms }]+3 \lambda_{\widetilde{\gamma}} \Delta \mathcal{H}+c_{G} \lambda_{\widetilde{\gamma}} \partial_{\ell} \mathcal{G}^{\ell}-6 c_{S} \lambda_{\widetilde{\gamma}} \mathcal{S},
\end{aligned}
$$

and those of the $\widetilde{A}$-adjusted BSSN formulation are

$$
\begin{aligned}
\partial_{t} \mathcal{H} & =[\text { Original Terms }], \\
\partial_{t} \mathcal{M}_{i} & =[\text { Original Terms }]+(1 / 2) \kappa_{A} \Delta \mathcal{M}_{i}, \\
\partial_{t} \mathcal{G}^{i} & =[\text { Original Terms }], \\
\partial_{t} \mathcal{A} & =[\text { Original Terms }]+\kappa_{A} \delta^{i j} \partial_{i} \mathcal{M}_{j}, \\
\partial_{t} \mathcal{S} & =[\text { Original Terms }],
\end{aligned}
$$

where $\Delta$ is the Laplacian operator in flat space. "Original Terms" refers to the right-hand side of the constraint propagation equations for the standard BSSN formulation. Full expressions for the terms are given in the appendix of [22].

\section{Appendix C: Constraint Propagation Equations of Standard BSSN Formulation with $\beta^{i}=0$}

The constraint propagation equations for the standard BSSN formulation with $\beta^{i}=0$ are as follows (the full expressions are available in the appendix of [22]).

$$
\begin{aligned}
\partial_{t} \mathcal{H}= & {[(2 / 3) \alpha K+(2 / 3) \alpha \mathcal{A}] \mathcal{H}+\left[-4 e^{-4 \varphi} \alpha\left(\alpha_{k} \varphi\right) \widetilde{\gamma}^{k j}-2 e^{-4 \varphi}\left(\partial_{k} \alpha\right) \widetilde{\gamma}^{j k}\right] \mathcal{M}_{j} } \\
+ & {\left[-2 \alpha e^{-4 \varphi} \widetilde{A}^{k}{ }_{j} \partial_{k}-\alpha e^{-4 \varphi}\left(\partial_{j} \widetilde{A}_{k \ell}\right) \widetilde{\gamma}^{k \ell}-e^{-4 \varphi}\left(\partial_{j} \alpha\right) \mathcal{A}\right] \mathcal{G}^{j} } \\
+ & {\left[2 \alpha e^{-4 \varphi} \widetilde{\gamma}^{-1} \widetilde{\gamma}^{\ell k}\left(\partial_{\ell} \varphi\right) \mathcal{A} \partial_{k}+(1 / 2) \alpha e^{-4 \varphi} \widetilde{\gamma}^{-1}\left(\partial_{\ell} \mathcal{A}\right) \widetilde{\gamma}^{\ell k} \partial_{k}+(1 / 2) e^{-4 \varphi} \widetilde{\gamma}^{-1}\left(\partial_{\ell} \alpha\right) \widetilde{\gamma}^{\ell k} \mathcal{A} \partial_{k}\right] \mathcal{S} } \\
+ & {\left[(4 / 9) \alpha K \mathcal{A}-(8 / 9) \alpha K^{2}+(4 / 3) \alpha e^{-4 \varphi}\left(\partial_{i} \partial_{j} \varphi\right) \widetilde{\gamma}^{i j}+(8 / 3) \alpha e^{-4 \varphi}\left(\partial_{k} \varphi\right)\left(\partial_{\ell} \widetilde{\gamma}^{\ell k}\right)+\alpha e^{-4 \varphi}\left(\partial_{j} \widetilde{\gamma}^{j k}\right) \partial_{k}\right.} \\
& +8 \alpha e^{-4 \varphi} \widetilde{\gamma}^{j k}\left(\partial_{j} \varphi\right) \partial_{k}+\alpha e^{-4 \varphi} \widetilde{\gamma}^{j k} \partial_{j} \partial_{k}+8 e^{-4 \varphi}\left(\partial_{\ell} \alpha\right)\left(\partial_{k} \varphi\right) \widetilde{\gamma}^{\ell k}+e^{-4 \varphi}\left(\partial_{\ell} \alpha\right)\left(\partial_{k} \widetilde{\gamma}^{\ell k}\right)+2 e^{-4 \varphi}\left(\partial_{\ell} \alpha\right) \widetilde{\gamma}^{\ell k} \partial_{k} \\
& \left.+e^{-4 \varphi} \widetilde{\gamma}^{\ell k}\left(\partial_{\ell} \partial_{k} \alpha\right)\right] \mathcal{A},
\end{aligned}
$$




$$
\begin{aligned}
& \partial_{t} \mathcal{M}_{i}=\left[-(1 / 3)\left(\partial_{i} \alpha\right)+(1 / 6) \partial_{i}\right] \mathcal{H}+\alpha K \mathcal{M}_{i}+\left[\alpha e^{-4 \varphi} \widetilde{\gamma}^{k m}\left(\partial_{k} \varphi\right)\left(\partial_{j} \widetilde{\gamma}_{m i}\right)-(1 / 2) \alpha e^{-4 \varphi} \widetilde{\Gamma}_{k \ell}^{m} \widetilde{\gamma}^{k \ell}\left(\partial_{j} \widetilde{\gamma}_{m i}\right)\right. \\
& +(1 / 2) \alpha e^{-4 \varphi} \widetilde{\gamma}^{m k}\left(\partial_{k} \partial_{j} \widetilde{\gamma}_{m i}\right)+(1 / 2) \alpha e^{-4 \varphi} \widetilde{\gamma}^{-2}\left(\partial_{i} \mathcal{S}\right)\left(\partial_{j} \mathcal{S}\right)-(1 / 4) \alpha e^{-4 \varphi}\left(\partial_{i} \widetilde{\gamma}_{k \ell}\right)\left(\partial_{j} \widetilde{\gamma}^{k \ell}\right) \\
& +\alpha e^{-4 \varphi} \widetilde{\gamma}^{k m}\left(\partial_{k} \varphi\right) \widetilde{\gamma}_{j i} \partial_{m}+\alpha e^{-4 \varphi}\left(\partial_{j} \varphi\right) \partial_{i}-(1 / 2) \alpha e^{-4 \varphi} \widetilde{\Gamma}_{k \ell}^{m} \widetilde{\gamma}^{k l} \widetilde{\gamma}_{j i} \partial_{m}+\alpha e^{-4 \varphi} \widetilde{\gamma}^{m k} \widetilde{\Gamma}_{i j k} \partial_{m} \\
& \left.+(1 / 2) \alpha e^{-4 \varphi} \widetilde{\gamma}^{\ell k} \widetilde{\gamma}_{j i} \partial_{k} \partial_{\ell}+(1 / 2) e^{-4 \varphi} \widetilde{\gamma}^{m k}\left(\partial_{j} \widetilde{\gamma}_{i m}\right)\left(\partial_{k} \alpha\right)+(1 / 2) e^{-4 \varphi}\left(\partial_{j} \alpha\right) \partial_{i}+(1 / 2) e^{-4 \varphi} \widetilde{\gamma}^{m k} \widetilde{\gamma}_{j i}\left(\partial_{k} \alpha\right) \partial_{m}\right] \mathcal{G}^{j} \\
& +\left[-\widetilde{A}^{k}{ }_{i}\left(\partial_{k} \alpha\right)+(1 / 9)\left(\alpha_{j}\right) K+(4 / 9) \alpha\left(\partial_{i} K\right)+(1 / 9) \alpha K \partial_{i}-\alpha \widetilde{A}^{k}{ }_{i} \partial_{k}\right] \mathcal{A}, \\
& \partial_{t} \mathcal{G}^{i}=2 \alpha \widetilde{\gamma}^{i j} \mathcal{M}_{j}+\left[4 \alpha \widetilde{\gamma}^{i j}\left(\widetilde{D}_{j} \varphi\right)-\alpha \widetilde{\gamma}^{i j} \partial_{j}-\left(\partial_{k} \alpha\right) \widetilde{\gamma}^{i k}\right] \mathcal{A}, \\
& \partial_{t} \mathcal{A}=\alpha K \mathcal{A} \text {, } \\
& \partial_{t} \mathcal{S}=-2 \alpha \widetilde{\gamma} \mathcal{A} .
\end{aligned}
$$

[1] R. Arnowitt, S. Deser, and C. W. Misner, in Gravitation: An Introduction to Current Research, edited by L. Witten (Wiley, New York, 1962).

[2] J. W. York, Jr., in Sources of Gravitational Radiation, edited by L. Smarr (Cambridge, 1979); L. Smarr and J. W. York, Jr., Phys. Rev. D 17, 2529 (1978).

[3] H. Shinkai and G. Yoneda, Classical Quantum Gravity 17, 4799 (2000).

[4] H. Shinkai and G. Yoneda, gr-qc/0209111 (2002).

[5] H. Shinkai, J. Korean Phys. Soc. 54, 2513 (2009).

[6] M. Shibata and T. Nakamura, Phys. Rev. D 52, 5428 (1995).

[7] T. W. Baumgarte and S. L. Shapiro, Phys. Rev. D 59, 024007 (1998).

[8] F. Pretorius, Classical Quantum Gravity 22, 425 (2005).

[9] D. Garfinkle, Phys. Rev. D 65, 044029 (2002).

[10] L. E. Kidder, M. A. Scheel, and S. A. Teukolsky, Phys. Rev. D 64, 064017 (2001).

[11] C. Bona, T. Ledvinka, C. Palenzuela, and M. Žáček, Phys. Rev. D 67, 104005 (2003).

[12] C. Bona, T. Ledvinka, C. Palenzuela, and M. Žáček, Phys. Rev. D 69, 064036 (2004).

[13] M. Campanelli, C. O. Lousto, P. Marronetti, and Y. Zlochower, Phys. Rev. Lett. 96, 111101 (2006).

[14] J. G. Baker, J. Centrella, D.-I. Choi, M. Koppitz, and J. van Meter, Phys. Rev. Lett. 96, 111102 (2006).

[15] F. Pretorius, Phys. Rev. Lett. 95, 121101 (2005).

[16] M. A. Scheel, M. Boyle, T. Chu, L. E. Kidder, K. D. Matthews, and H. P. Pfeiffer, Phys. Rev. D 79, 024003 (2009).
[17] D. Alic, C. Bona-Casas, C. Bona, L. Rezzolla, and C. Palenzuela, gr-qc/1106.2254 (2011).

[18] C. Gundlach, G. Calabrese, I. Hinder, and J. M. MartínGarcía, Classical Quantum Gravity 22, 3767 (2005).

[19] A. Weyhausen, S. Bernuzzi, and D. Hilditch, grqc/1107.5539 (2011).

[20] G. Yoneda and H. Shinkai, Phys. Rev. D 63, 124019 (2001).

[21] H. Shinkai and G. Yoneda, Classical Quantum Gravity 19, 1027 (2002).

[22] G. Yoneda and H. Shinkai, Phys. Rev. D 66, 124003 (2002).

[23] K. Kiuchi and H. Shinkai, Phys. Rev. D 77, 044010 (2008).

[24] D. R. Fiske, Phys. Rev. D 69, 047501 (2004).

[25] D. R. Fiske, Ph.D. thesis, University of Maryland, College Park (2004).

[26] T. Tsuchiya, G. Yoneda, and H. Shinkai, Phys. Rev. D 83, 064032 (2011).

[27] M. Alcubierre et al., Classical Quantum Gravity 21, 589 (2004).

[28] G. Zumbusch, Classical Quantum Gravity 26, 175011 (2009).

[29] C. Bona and C. Bona-Casas, Phys. Rev. D 82064008 (2010).

[30] V. Paschalidis, J. Hansen, and A. Khokhlov, Phys. Rev. D 78, 064048 (2008).

[31] S. Bernuzzi and D. Hilditch, Phys. Rev. D 81084003 (2010). 